\title{
A Rough Set-Based Magnetic Resonance Imaging Partial Volume Detection System
}

\author{
Sebastian Widz ${ }^{1,2}$, Kenneth Revett ${ }^{3}$, and Dominik Ślęzak ${ }^{4,2}$ \\ 1 Deforma Sebastian Widz, Warsaw, Poland \\ 2 Polish-Japanese Institute of Information Technology, Warsaw, Poland \\ 3 University of Westminster, Harrow School of Computer Science, London, UK \\ 4 Department of Computer Science, University of Regina, Regina, Canada
}

\begin{abstract}
Segmentation of magnetic resonance imaging (MRI) data entails assigning tissue class labels to voxels. The primary source of segmentation error is the partial volume effect (PVE) which occurs most often with low resolution imaging - With large voxels, the probability of a voxel containing multiple tissue classes increases. Although the PVE problem has not been solved, the first stage entails correctly identifying PVE voxels. We employ rough sets to identify them automatically.
\end{abstract}

\section{Introduction}

Image segmentation is the process of assigning the class labels to data containing spatially varying information. In case of Magnetic Resonance Imaging (MRI) of the brain, segmentation relies on the magnitude values of voxels collected during the scanning procedure [6, 13. The brain is imaged through the $2 \mathrm{D}$ slices sampled at a particular thickness. The goal is to classify every voxel within a slice to one of the tissue classes. It has been demonstrated that their relative distribution is diagnostic for specific diseases [3, 4].

Among many difficulties in segmenting MRI data, there is the partial volume effect (PVE) 2, 9]. In this paper, we focus on the automated detection of voxels subject to PVE. mthods developed for this problem so far do not provide satisfactory solution [1,7]. We consider the theory of rough sets [8, particularly the approach based on approximate reducts 11,12, as an alternative tool. Results are obtained for the data from Simulated Brain Database (SBD) 1 . They can be incorporated with our previous work on MRI segmentation [14, 15].

\section{Data Preparation}

The magnitudes of voxels are given in three modalities (T1, T2, PD). Under normal circumstances, the magnitudes of voxels form Gaussian distributions corresponding to the following tissue classes: bone and background (BCG), Cerebral Spinal Fluid (CSF), Grey Matter (GM), White Matter (WM), and others (fat, skin, muscle), as visible Figure 1 We focus on CSF, GM, and WM.

\footnotetext{
${ }^{1}$ SBD database is provided by the Brain Imaging Centre, Montreal Neurological Institute http://www.bic.mni.mcgill.ca/brainweb
} 


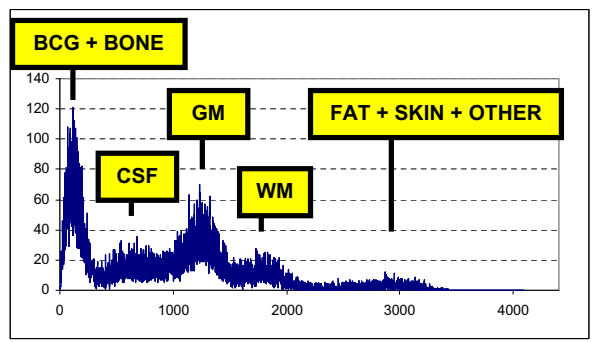

Fig. 1. The magnitude histogram obtained from a T1 slice. The $\mathrm{x}$-axis corresponds to the magnitude of voxels. The peaks are likely to correspond to particular tissue classes.

To develop the PVE identification procedure, we first generate the training data. Following the standards of the theory of rough sets [5, 8, we form a decision table $\mathbb{A}=(U, A \cup\{d\})$, where each attribute $a \in A \cup\{d\}$ is a function $a: U \rightarrow V_{a}$ from the universe $U$ of voxels into the value set $V_{a}$. The elements of $U$ are voxels. The set $A$ contains the following binary attributes extracted for each modality:

EDGE attributes are derived by a modified Discrete Laplacian method - a non-directional gradient operator determining whether neighborhood of a voxel is homogenous within a specified tolerance. We use a threshold for homogeneity tuned separately using the T1, T2, and PD training images. We consider this attribute type because voxels on tissue edges are more likely to suffer from PVE. We put the voxel's value as 1 in case inhomogeneity is detected and 0 otherwise.

MAGNITUDE attributes are derived using the magnitude histogram clustering 14. Its idea is to produce clusters as intervals around the histogram's peaks and within the gaps. The distance between the magnitude levels is illustrated by Figure 2. The algorithm is controlled by parameters deciding about the number of peaks detected and size of intervals around them, which are tuned as in case of the edge attributes. Since the histogram's peaks are likely to correspond to particular tissues, we define this attribute's value as 1 for voxels with magnitudes within the peak intervals and 0 for those dropping into the gaps.

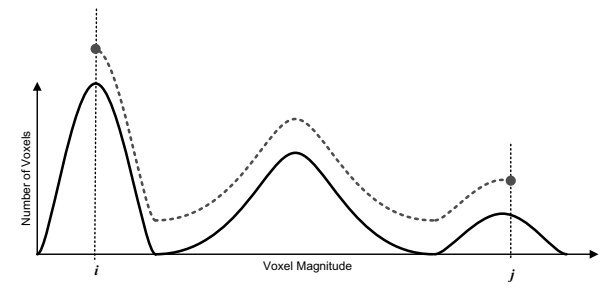

Fig. 2. Illustration of the histogram distance between magnitude positions $i$ and $j$

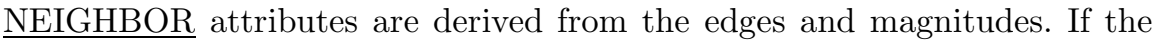
edge is not detected for a given voxel, the neighbor attribute's value is copied from the magnitude attribute. Otherwise, it is equal to the magnitude attribute's value (0 or 1 ) occurring more frequently within the direct voxel's neighborhood. 


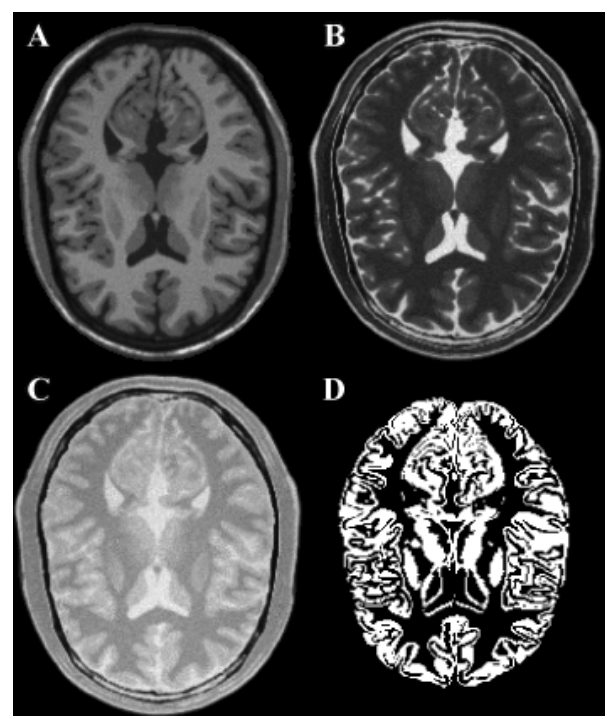

Fig. 3. Modalities T1 (A), T2 (B), and PD (C). D presents PVE (black) and NOE (white) decision classes obtained from the fuzzy SBD phantom for the same slice.

Attribute $d \notin A$ indicates whether voxels belong to PVE or NOE (NO-Effect) decision classes. For training and evaluation of the PVE identification accuracy, it is derived from so called fuzzy phantoms taken from SBD data, where each voxel is labeled with memberships to particular tissue types. We use the threshold to decide whether a given voxel belongs to the NOE class (if its membership to one of tissues exceeds enough the average tissue content level for the given slice) or to PVE class (if memberships are not diversified enough to decide). The threshold is tuned over the middle brain slice to get approximately 30\%/70\% distribution between the PVE and NOE classes, as illustrated by Figure 3 .

\section{Attribute Tuning and Reduction}

While classifying new cases, one must strike a balance between accuracy and computational complexity. It can be achieved through the use of a decision reduct [5, 8]: an irreducible subset $B \subseteq A$ determining $d$ in decision table $\mathbb{A}=(U, A \cup$ $\{d\})$. Reducts are used to produce the decision rules from the training data. Sometimes it is better to remove more attributes to get shorter rules at the cost of their slight inconsistencies. Let us consider the following measure:

$$
R(d / B)=\sum_{\text {rules } r \text { induced by } B}\left(\frac{\text { number of objects recognizable by } r}{\text { number of objects in } U} *\right.
$$

$$
\left.\max _{i} \frac{\text { probability of the } i \text {-th decision class induced by } r}{\text { prior probability of the } i \text {-th decision class }}\right)
$$




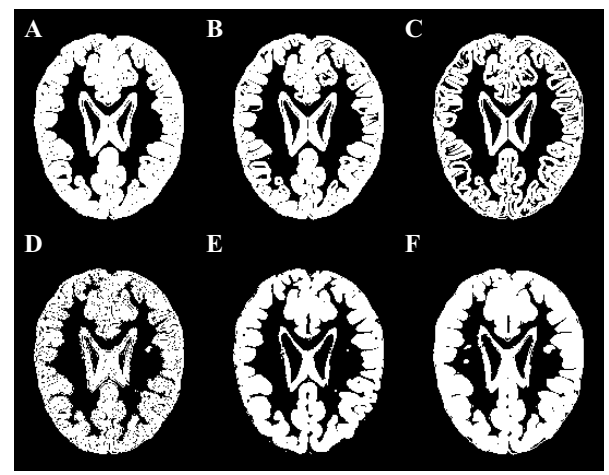

Fig. 4. Attributes derived for a single slice from the phantom data set. Image A corresponds to the edge attribute based on modality $\mathrm{T} 1$. $\mathrm{B}$ and $\mathrm{C}$ correspond to $\mathrm{T} 2$ and $\mathrm{PD}$, respectively. The second row of images corresponds to the magnitude attributes.

Measure $R$ expresses the average gain in determining decision classes under the evidence provided by the rules generated by $B \subseteq A$ 11, 12. Given approximation threshold $\varepsilon \in[0,1)$, let us say that $B \subseteq A$ is an $(R, \varepsilon)$-approximate decision reduct, if and only if it satisfies inequality $R(d / B) \geq(1-\varepsilon) * R(d / A)$ and none of its proper subsets does it. We derive such approximate reducts from the MRI-related training data described in the previous section, using the orderbased hybrid genetic algorithm adapted from [10,16]. The results of applying the reduct-based rules to the testing images are presented in the next section.

$R$ can be used also to evaluate single attributes. In particular, we apply it to tune the previously-mentioned parameters of the edge and magnitude attributes. The resulting attributes approximate very well the less frequent and actually more important PVE decision class - its area is almost perfectly contained in the borderline regions indicated by attributes $a \in A$ maximizing $R(d /\{a\})$. Examples of such obtained regions are illustrated by Figure 4. Consequently, the PVE identification procedure based on such attributes is expected to avoid misclassifying the PVE voxels as the ones which do not suffer from PVE.

\section{Preliminary Results}

The SBD phantoms have a complete set of MRI volumes, including partial voxel volumes of varying slice thickness $(3-9 \mathrm{~mm})$. For this study, we employed the $3 \mathrm{~mm}$ thick volumes. We selected a range of slices from the center of the volume (27-36) for training/testing purposes. Reducts and rules were generated from the training set. The results are presented in Figure 5. For high $\varepsilon \in[0,1)$, the reducts are empty, leading to a blind guess classification of all voxels to the larger class (no partial volume effect). In practice, however, we are more interested in sensitivity (accurate recognition of PVE), even if it leads to an increase of the false positive fraction. The accuracy curve suggests that for intermediate approximation thresholds the obtained non-empty reducts still provide a reasonable overall score and may better approximate PVE by decision rules. 


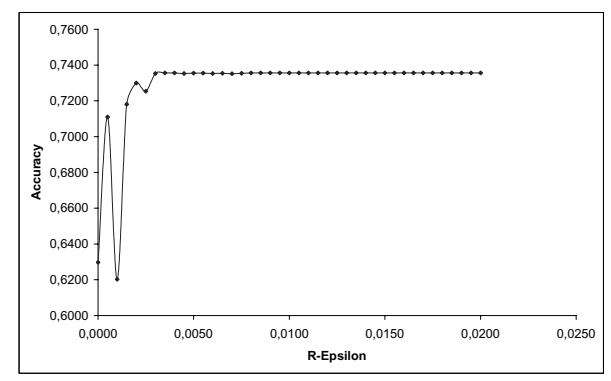

Fig. 5. Average accuracy of PVE identification with respect to the choice of $\varepsilon \in[0,1)$

In Figure 6 it is visible that the shape of PVE classified area matches the actual one. Moreover, our method provides valuable results targeted at better PVE sensitivity and specificity. The amount of misclassified PVE voxels is considerably smaller than for NOE. Further research is needed to improve overall accuracy.

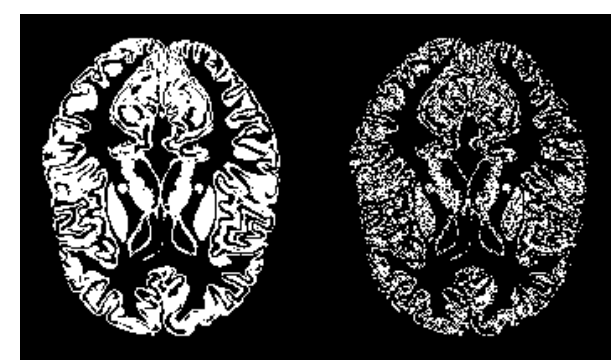

\begin{tabular}{|c|c|c|c|c|c|}
\hline Slice no. 27 & NOE & PVE & Slice no. 36 & NOE & $P V$ \\
\hline Actual NOE & 7106 & 6768 & Actual NOF & 7197 & 5198 \\
\hline Actual PVE & 1277 & 5099 & Actual PVE & 976 & \\
\hline
\end{tabular}

Fig. 6. Example of PVE-identification for intermediate $\varepsilon \in[0,1)$. Top left: The actual PVE area (black) taken from the phantom. Top right: the result of classification. Below: Confusion matrices for two other testing slices, obtained using non-empty reducts.

\section{Conclusion}

We presented a rough set-based system for partial volume voxel detection in multi-modal MR images. To extract relevant attributes, we utilized the edge detection and magnitude clustering methods on three imaging modalities. Decisions were obtained from fuzzy phantoms, taken from Simulated Brain Database. The obtained preliminary results show that the proposed method, based on simple binary attributes, is worth further development. Our approach forms the basis of a comprehensive automated segmentation algorithm. First, voxels not subject to PVE may be identified and segmented using previously developed methodology [14,15]. The remaining voxels, presumed to suffer from PVE, may be processed 
accordingly. It provides the first instance of a rough set system that is capable of segmenting MR images regardless of occurrence of PVE.

Acknowledgments. The research reported in this article was supported in part by research grants from Natural Sciences and Engineering Research Council of Canada awarded to the third author.

\section{References}

1. Aston, J.A.D., Cunningham, V.J., Asselin, M.-C., Hammers, A., Evans, A.C., Gunn, R.N.: Positron emission tomography partial volume correction: estimation and algorithms. J Cereb Blood Flow Metab 22 (2002) 1019-1034

2. Atkins, M.S., Mackiewich, B.T.: Fully Automatic Segmentation of the Brain in MRI. IEEE Transcations on Medical Imaging 17(1) (1998)

3. Kamber, M., Shinghal, R., Collins, L.: Model-based 3D Segmentation of Multiple Sclerosis Lesions in Magnetic Resonance Brain Images. IEEE Trans Med Imaging 14(3) (1995) 442-453

4. Kaus, M., Warfield, S.K., Nabavi, A., Black, P.M., Jolesz, F.A., Kikinis, R.: Automated Segmentation of MRI of Brain Tumors. Radiology 218 (2001) 586-591

5. Komorowski, J., Pawlak, Z., Polkowski, L., Skowron, A.: Rough sets: A tutorial. In: S.K. Pal, A. Skowron (eds): Rough Fuzzy Hybridization - A New Trend in Decision Making. Springer Verlag (1999) 3-98

6. Kovacevic, N., Lobaugh, N.J., Bronskill, M.J., Levine, B., Feinstein, A., Black, S.E.: A Robust Extraction and Automatic Segmentation of Brain Images. NeuroImage 17 (2002) 1087-1100

7. Matsuda, H., Ohnishi, T., Asada, T., Li, Z., Kanetaka, H., Imabayashi, E., Tanaka, F., Nakano, S.: Correction for Partial-Volume Effects on Brain Perfusion SPECT in Healthy Men. J Nucl Med. 44 (2003) 1243-1252

8. Pawlak, Z.: Rough sets - Theoretical aspects of reasoning about data. Kluwer Academic Publishers (1991)

9. Pham, D.L., Prince, J.L.: Unsupervised partial volume estimation in single-channel imaging data. In: Proc MMBIA'00. Hilton Head Island, SC (2000)

10. Ślęzak, D., Wróblewski, J.: Order-based genetic algorithms for the search of approximate entropy reducts. In: Proc RSFDGrC'2003. Chongqing, China (2003)

11. Ślęzak, D., Ziarko, W.: Attribute Reduction in Bayesian Version of Variable Precision Rough Set Model. In: Proc. of RSKD'2003. Elsevier, ENTCS 82(4) (2003)

12. Ślęzak, D., Ziarko, W.: The Investigation of the Bayesian Rough Set Model. International Journal of Approximate Reasoning (2005)

13. Van Leemput, K., Maes, F., Vandermeulen, D., Colchester, A., Suetens, P.: Automated model-based tissue classification of MR images on the brain. IEEE Trans Med. Imag. 18 (1999) 897-908

14. Widz, S., Revett, K., Ślęzak, D.: A Hybrid Approach to MR Image Segmentation Using Unsupervised Clustering and Approximate Reducts. In: Proc RSFDGrC'2005. Regina, Canada (2005)

15. Widz, S., Ślȩzak, D., Revett, K.: An Automated Multi-spectral MRI Segmentation Algorithm Using Approximate Reducts. In: Proc RSCTC'2004. Uppsala, Sweden (2004)

16. Wróblewski, J.: Theoretical Foundations of Order-Based Genetic Algorithms. Fundamenta Informaticae 28(3-4) (1996) 423-430 